\title{
Optimization of pulsed ultrasound-assisted technique for extraction of phenolics from pomegranate peel of Malas variety: punicalagin and hydroxybenzoic acids
}

\begin{abstract}
Pomegranate peel is a rich source of phenolic compounds (such as punicalagin and hydroxybenzoic acids). However, the content of such bioactive compounds in the peel extract can be affected by extraction type and condition. It was hypothesized that the optimization of a pulsed ultrasound-assisted extraction (PUAE) technique could result in the pomegranate peel extract with higher yield and antioxidant activity. The main goal was to optimize PUAE condition resulting in the highest yield and antioxidant activity as well as the highest contents of punicalagin and hydroxybenzoic acids. The operation at the intensity level of $105 \mathrm{~W} / \mathrm{cm} 2$ and duty cycle of $50 \%$ for a short time $(10 \mathrm{~min})$ had a high efficiency for extraction of phenolics from pomegranate peel. The application of such short extraction can save the energy and cost of the production. Punicalagin and ellagic acid were the most predominant phenolic compounds quantified in the pomegranate peel extract (PPE) from Malas variety. PPE contained a minor content of gallic acid.
\end{abstract}

Keyword: Pomegranate peel extract (PPE); Punicalagin; Hydroxybenzoic acids; Antioxidant activity; Pulsed ultrasound-assisted extraction 